\title{
Tau neuropathology correlates with FDG-PET, but not AV-1451-PET, in progressive supranuclear palsy
}

\author{
Ruben Smith $^{1,2}$ (1) Michael Schöll ${ }^{2,3} \cdot$ Michael Honer $^{4} \cdot$ Christer F. Nilsson $^{1,2}$ • \\ Elisabet Englund ${ }^{5}$ Oskar Hansson ${ }^{2,6}$
}

Received: 24 October 2016 / Revised: 14 November 2016 / Accepted: 24 November 2016 / Published online: 29 November 2016

(C) The Author(s) 2016. This article is published with open access at Springerlink.com

Radiotracers for tau have recently become available for positron emission tomography (PET) studies in neurodegenerative disorders. The currently most utilized tracer ${ }^{18} \mathrm{~F}$ AV-1451 binds to paired helical filaments containing both $3 R$ and $4 R$ tau in Alzheimer's disease (AD) in vitro [3, 4, 6]. Furthermore, ${ }^{18} \mathrm{~F}-\mathrm{AV}-1451$ can quantify AD-like tau pathology in MAPT R406W mutation carriers in vivo [7]. In corticobasal degeneration, there is a clear binding to $4 \mathrm{R}$ tau pathology, but SUVRs are generally lower than in AD $[2,5]$.

Whether the tracer binds to the straight filament $4 \mathrm{R}$ pathology in progressive supranuclear palsy (PSP) is not fully established. Autoradiographic studies $[3,4,6,8]$ have

Electronic supplementary material The online version of this article (doi:10.1007/s00401-016-1650-1) contains supplementary material, which is available to authorized users.

Ruben Smith

Ruben.Smith@med.lu.se

Oskar Hansson

Oskar.Hansson@med.lu.se

1 Department of Neurology, Skåne University Hospital, 20502 Malmö, Sweden

2 Clinical Memory Research Unit, Department of Clinical Sciences Malmö, Lund University, Lund, Sweden

3 Department of Clinical Neuroscience, MedTech West and the University of Gothenburg, Gothenburg, Sweden

4 Roche Pharmaceutical Research and Early Development, Neuroscience Discovery and Biomarkers, Roche Innovation Center Basel, Basel, Switzerland

5 Division of Oncology and Pathology, Department of Clinical Sciences Lund, Lund University, Lund, Sweden

6 Memory Clinic, Skåne University Hospital, 20502 Malmö, Sweden not shown convincing binding of AV-1451 to tau pathology in PSP. We have recently shown increased retention in vivo of ${ }^{18} \mathrm{~F}-\mathrm{AV}-1451$ in the basal ganglia, but not in the cortex, of patients with PSP [8]. Here, we present data from a 71-year-old male subject, who was diagnosed with PSP in 2011 after a 2-year history of increasing bradykinesia, rigidity, and falls (see Supplementary material for detail). Seven months prior to death the subject was scanned with ${ }^{18}$ F-AV-1451 PET, ${ }^{18}$ F-AV-FDG-PET, and MRI (Fig. 1a-d). ${ }^{18} \mathrm{~F}-\mathrm{AV}-1451$ PET showed retention in the basal ganglia, but not cortex (Fig. 1a, c). ${ }^{18} \mathrm{~F}-\mathrm{AV}$-FDG-PET revealed decreased metabolism in the frontal cortex and basal ganglia (Fig. 1b). Voxel-based morphometry showed cortical atrophy, predominantly in the frontal cortex (Fig. 1d). For methodological data, see the supplement. Post mortem neuropathological assessment confirmed the diagnosis of PSP, with a marked atrophy of the midbrain and a frontal cortical atrophy. Gallyas silver stain showed dense neurites and stained cell bodies in the basal ganglia, midbrain, and cerebral cortex, as well as cortical tufted astrocytes. Immunohistochemistry showed positivity for $4 \mathrm{R}$ tau inclusions, but no 3R tau staining (see supplement for method). Amyloid stains as well as $\alpha$-synuclein immunohistochemistry were negative (data not shown). Tau-positive (AT8 immunohistochemistry) neurites and cell bodies were quantified using unbiased stereology (see supplement and [7] for methods), and results were correlated with ${ }^{18} \mathrm{~F}-\mathrm{AV}-1451$ and ${ }^{18} \mathrm{~F}-\mathrm{FDG}$ retention in the same regions (Fig. 1 ). In the frontal, temporal, and parietal cortex and subcortical white matter, there was widespread tau pathology, whereas the occipital lobe and cerebellar grey matter were relatively spared (Fig. 1e-p). The basal ganglia and mesencephalon showed a widespread and intense tau pathology using immunohistochemistry. Comparing the ${ }^{18} \mathrm{~F}-\mathrm{AV}-1451$ retention to neurite density, there was no correlation between PET signal 

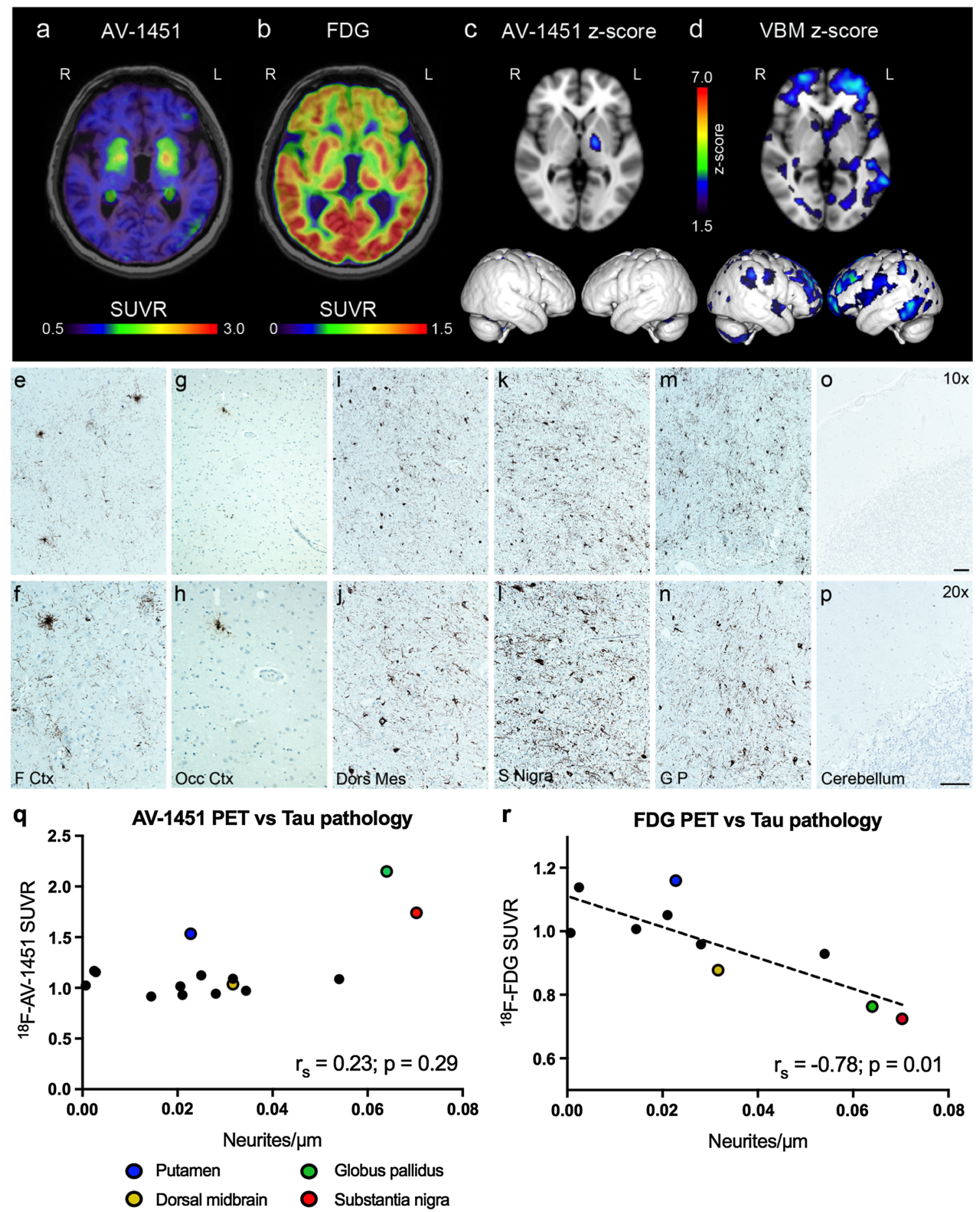

Fig. 1 PET retention and neuropathology. a SUVR PET image of AV-1451. b SUVR PET image of FDG. c Transversal and lateral view $\mathrm{z}$-score images of AV-1451 PET signal. d Transversal and lateral view z-score of atrophy maps based on inverted voxel-based morphometry. For both z-score images, pixels with values $>1.5$ (vs controls) are shown. AT8-immunohistochemistry at $\times 10$ (upper panel) or $\times 20$

(lower panel) magnification of frontal pole cortex $(\mathbf{e}, \mathbf{f})$, occipital cortex $(\mathbf{g}, \mathbf{h})$, dorsal midbrain $(\mathbf{i}, \mathbf{j})$, substantia nigra $(\mathbf{k}, \mathbf{l})$, globus pallidus $(\mathbf{m}, \mathbf{n})$, and cerebellar cortex $(\mathbf{o}, \mathbf{p})$. Scale bars $50 \mu \mathrm{m} . \mathbf{q}{ }^{18} \mathrm{~F}$ AV-1451 SUVRs plotted against the tau-positive neurite density. An annotated version of this figure is available as Supplementary Fig. 2. $\mathbf{r}^{18}$ F-FDG SUVRs plotted against neurite density 
and the intensity of the pathology $\left(r_{\mathrm{s}}=0.23 ; p=0.29\right.$; Fig. 1q). Notably, the highest standardized uptake value ratios (SUVRs) were seen in the globus pallidus, putamen, and substantia nigra, areas with previously reported off-target binding $[1,4,8]$. The cortical and white matter ${ }^{18} \mathrm{~F}-\mathrm{AV}-1451$ SUVRs were low $(<1.2)$ and showed no association with tau immunohistochemistry. Furthermore, autoradiography of AV-1451 and THK-5351 showed no specific binding to tau pathology (Supplementary Fig. 1). ${ }^{18}$ F-FDG retention in the cerebral cortex, brain stem, and basal ganglia showed a clear inverse correlation to tau neuropathology $\left(r_{\mathrm{s}}=-0.78 ; p=0.01 ;\right.$ Fig. $\left.1 \mathrm{r}\right)$.

In conclusion, the ${ }^{18} \mathrm{~F}-\mathrm{AV}-1451$ uptake in cerebral cortex and white matter does not reflect the tau pathology in PSP, possibly due to a less abundant tau pathology compared to AD and a lower affinity of AV-1451 for straight 4R filaments. In the basal ganglia and brain stem, where offtarget binding is strong, it is unclear whether the increased ${ }^{18} \mathrm{~F}-\mathrm{AV}-1451$ reflects true tau pathology, even though the absence of specific autoradiographic binding in these structures argues against this notion. However, an increase in tau pathology is clearly associated with a decrease in ${ }^{18}$ F-FDG-PET.

Acknowledgements We are grateful to the patient and his family for participating in the study. We also thank Drs. Tomas Björklund, Douglas Hägerström, Jonas Jögi, and Tomas Olsson. The study was supported by the European and the Swedish Research Councils. We are grateful to Avid radiopharmaceuticals for providing the precursor to $\mathrm{AV}-1451$.

Open Access This article is distributed under the terms of the Creative Commons Attribution 4.0 International License (http://creativecommons.org/licenses/by/4.0/), which permits unrestricted use, distribution, and reproduction in any medium, provided you give appropriate credit to the original author(s) and the source, provide a link to the Creative Commons license, and indicate if changes were made.

\section{References}

1. Hansen AK, Knudsen K, Lillethorup TP et al (2016) In vivo imaging of neuromelanin in Parkinson's disease using 18F-AV1451 PET. Brain. doi:10.1093/brain/aww098

2. Josephs KA, Whitwell JL, Tacik P et al (2016) [18F]AV-1451 tau-PET uptake does correlate with quantitatively measured 4R-tau burden in autopsy-confirmed corticobasal degeneration. Acta Neuropathol 132:931-933. doi:10.1007/ s00401-016-1618-1

3. Lowe VJ, Curran G, Fang P et al (2016) An autoradiographic evaluation of AV-1451 Tau PET in dementia. Acta Neuropathol Commun 4:58. doi:10.1186/s40478-016-0315-6

4. Marquie M, Normandin MD, Vanderburg CR et al (2015) Validating novel tau positron emission tomography tracer [F-18]-AV-1451 (T807) on postmortem brain tissue. Ann Neurol 78:787-800. doi:10.1002/ana.24517

5. McMillan CT, Irwin DJ, Nasrallah I et al (2016) Multimodal evaluation demonstrates in vivo $18 \mathrm{~F}-\mathrm{AV}-1451$ uptake in autopsy-confirmed corticobasal degeneration. Acta Neuropathol 132:935-937. doi:10.1007/s00401-016-1640-3

6. Sander K, Lashley T, Gami P et al (2016) Characterization of tau positron emission tomography tracer $[\mathrm{F}] \mathrm{AV}-1451$ binding to postmortem tissue in Alzheimer's disease, primary tauopathies, and other dementias. Alzheimers Dement. doi:10.1016/j. jalz.2016.01.003

7. Smith R, Puschmann A, Scholl M et al (2016) 18F-AV-1451 tau PET imaging correlates strongly with tau neuropathology in MAPT mutation carriers. Brain 139:2372-2379. doi:10.1093/ brain/aww163

8. Smith R, Schain M, Nilsson C et al (2016) Increased basal ganglia binding of $18 \mathrm{~F}-\mathrm{AV}-1451$ in patients with progressive supranuclear palsy. Mov Disord. doi:10.1002/mds.26813 